\title{
Commentary
}

\section{Groundwater Iron and the Basis for Microbiota- Directed Complementary Food Treatment of Child Undernutrition}

Sabuktagin Rahman ${ }^{1 *}$ M.B.B.S., M.Sc., M.F.S.N.; Santhia Ireen ${ }^{2}$ M.Sc., M.Phil., Ph.D.

${ }^{1}$ Public Health, School of Medicine, Griffith University, Gold Coast Campus, QLD 4222, Australia

${ }^{2}$ Alive and Thrive Bangladesh, Genetic Baro Bhuiyan, Kemal Ataturk Avenue, Gulshan 2, Dhaka-1212, Bangladesh

*Corresponding author.

E-mail: sabuktagin.rahman@griffithuni.edu.au;

Phone: +61 481873612

Conflicts of interest: The authors declare no competing interests.

\section{Childhood undernutrition: Is a novel solution imminent?}

Undernutrition affecting approximately 200 million under-five children is a prominent public health problem globally (UNICEF, 2019) and is considered a key indicator of child health inequalities (World Health Organization, 1995). Despite heightened efforts in various countries to curb this, the progress remains sluggish in many parts of the world. Among numerous efforts to address undernutrition, composition of gut microbiota is the newest proposition which is touted to address undernutrition by optimizing biological mechanisms of child growth and immunity (Robertson et al, 2020). A recent trial has generated huge interest among public health scientists, programmers and the donor community. We refer to the work of Gehrig et al. (2019). The study, conducted in Bangladesh, unlocks the potential of a novel way of addressing undernutrition in young children by using complementary foods favorable to the growth of healthy gut microbiota.

An element of postnatal human development involves formation of microbial communities in different body parts, including the gut. Children suffering from acute malnutrition have compromised development of their gut microbiota, rendering them with microbiota communities 
that appear younger (more immature) than those of age-matched healthy individuals. Interactions between therapeutic diet, microbiota development, and growth recovery have been studied in recent times. Microbiota-directed complementary foods (MDCFs) are the ingredients of complementary foods identified using animal models to nudge the microbiota into a mature postweaning state, with potential to support child growth (Gehrig et al, 2019). The Gehrig et al study developed the MDCFs that enhance the representation of beneficial functions of the growthpromoting bacterial taxa in the developing microbiota. Among the devised MDCFs, one (MDCF2) was most effective in repairing the gut microbiota towards the healthy-state. This enabled greater levels of the biomarkers and mediators of child growth, bone formation, neurodevelopment and immune function in 12-18 months old undernourished children compared to the standard treatment.

\section{Groundwater iron - a natural source of iron from drinking water improving iron status in populations}

Groundwater iron was not considered in the above study which has been an emerging environmental factor demonstrating a positive association with iron sufficiency in populations in Bangladesh and in other South Asian countries (Rahman et al, 2016; Karakochuk et al, 2015). This is a natural remedy against iron-deficiency in many settings where the traditional diet is inadequate with iron (Rahman et al, 2016). Groundwater is the principal source of potable supply, and an estimated $97 \%$ of Bangladeshi population depend on this for drinking water (British Geological Survey 2001). People usually prefer cool, freshly pumped groundwater habitually which is mostly untreated. The concentration of iron in groundwater is predominantly high in many parts of the country (British Geological Survey 2001).

\section{Nexus of supplemental iron/iron intake and gut microbiota- induced side-effects}

Iron, while essential for some key physiological functions and cognitive development of children may cause side effects if there is an excess in the body; and this is mediated through ironinduced adversaries in the composition of gut microbiota (Jaeggi et al, 2015). Trials in African children have shown that supplemental iron with an array of different doses (2.5 -20.0 mg/day) resulted in adverse effects on the composition of gut microbiota compared to the placebo--e.g., higher relative abundance and virulence of disease-causing Salmonella, Shigella or pathogenic Escherichia coli and a lower abundance of health-promoting beneficial bacteriae.g. Bifidobacteriaceae, Lactobacillaceae (Jaeggi et al, 2015; Zimmermann et al, 2010). These studies also documented increased level of intestinal inflammation as shown by higher levels of fecal calprotectin in the supplemented children. In Bangladesh, no study has been conducted to document the effect of excess iron in drinking water/supplemental iron on the composition of gut microbiota. However, anecdotal experience suggests that in the high groundwater iron areas, people commonly experience abdominal discomfort with occasional loose stools. A recent trial on controlling anemia in Bangladeshi children residing in a high groundwater iron setting 
(groundwater iron concentration $\geq 2 \mathrm{mg} / \mathrm{L}$ ), compared the efficacy and side effects of an MNP (micronutrient powder) with a low-dose of iron (5 mg iron as ferrous fumarate) against the standard MNP (containing $12.5 \mathrm{mg}$ iron). The trial reported significantly lower incidences of side effects such as diarrhea, nausea, and fever in children receiving the low-iron MNP (Rahman et al, 2019).

\section{High groundwater iron and potential implications for the MDCF's effects on child undernutrition}

The site of the Gehrig et al study (Gehrig et al, 2019) is the capital city Dhaka, where iron content in groundwater is negligible (British Geological Survey, 2001). Hence, the amount of iron in the study children acquired from drinking water could be trace, if any. However, in the high-iron groundwater areas which constitute roughly half of the country (Rahman et al, 2016), a study observed that the amount of iron acquired from drinking groundwater alone was 50\% higher than that acquired from the diet in 2-5-year-old children (Rahman et al, 2019).

Absorption of iron is inversely correlated to the amount of the reserve of body iron, mediated through hepcidin-induced regulation (Saito et al, 2014). National prevalence of iron deficiency in under-five children in Bangladesh is low (10.7\%). Mean national estimates of the infectionadjusted ferritin in children are $38.9 \pm 1.9$ and $23.1 \pm 1.9 \mathrm{ng} / \mathrm{ml}$ in the predominantly high-iron and predominantly low-iron groundwater areas respectively; which are a few folds higher than the cut-off defining iron deficiency (ferritin< $12 \mathrm{ng} / \mathrm{ml})$ ((Rahman et al, 2016).

Hence, with a fair level of the iron reserve in the population (Rahman et al, 2016), a high amount of ongoing daily ingestion of iron from drinking groundwater may activate the regulation of absorption, resulting in unabsorbed iron in the intestines. This may plausibly cause independent adversaries on composition of gut microbiota, i.e., increasing and decreasing the relative abundance of the disease-causing and health promoting bacteria respectively. These adversaries due to high level of groundwater iron (iron from supplementation programs for prevention of anemia may additionally be present) may conceivably tamper with and/or offset the MDCFinduced effects of the promotion of the children's growth-promoting healthy microbiota.

The site of the study (Gehrig et al, 2019) (a slum in the capital city, Dhaka) is unrepresentative of the country; and thus, authors indicated that further studies would be conducted on a larger scale. If the study is replicated in a high groundwater iron setting (iron concentration of the children's drinking water $\geq 2 \mathrm{mg} / \mathrm{L}$ ); and accounts for intake of iron from drinking groundwater, it would be interesting to observe if its findings in children--the effect of the MDCFs on rejuvenation of healthy microbiota; and the promotion of the biomarkers and the mediators of child growth and development-apply in other settings.

This is important to confirm the probable health benefits of MDCFs in preventing child undernutrition in representative populations of Bangladesh; and potentially at a global scale as some 2 billion people mostly in the low- and middle-income countries rely on groundwater as their principal potable supply (Graaf et al, 2016). Testing the efficacy of the MDCFs in iron- 
replete population living on groundwater naturally rich in iron as potable supply is critically important. The outcome would complement the evaluation of MDCF's credentials as a novel solution of child undernutrition.

\section{References}

1. United Nations Children's Fund (UNICEF), 2019. The State Of The World's Children, A fair chance for every child. New York: UNICEF.

2. World Health Organization (WHO), 1995. Physical status: the use and interpretation of anthropometry - Report of a WHO Expert Committee. Geneva

3. Robertson RC, 2020. The Gut Microbiome in Child Malnutrition. In: Michaelsen KF, Neufeld LM, Prentice AM (eds): Global Landscape of Nutrition Challenges in Infants and Children. Nestlé Nutr Inst Workshop Ser 93, 133-144. Nestlé Nutrition Institute, Switzerland/S. Karger AG, Basel.

4. Gehrig JL, Venkatesh S, Chang H, Hibberd MC, Kung VL, Cheng J, et al., 2019. Effects of microbiota-directed foods in gnotobiotic animals and undernourished children. Science 365, (6449), eaau4732. DOI: 10.1126/science.aau4732

5. Rahman S, Ahmed T, Rahman AS, Alam N, Ahmed AMS, Ireen S, Chowdhury IA, Rahman SMM, 2016. Determinants of iron status and Hb in the Bangladesh population: The role of groundwater iron. Public Health Nutr, 19, 1862-187.

6. Karakochuk CD, Murphy HM, Whitfield KC, Barr SI, Vercauteren SM, Talukder A, Porter K, Kroeun H, Eath M, McLean J, and Green TJ, 2015. Elevated levels of iron in groundwater in Prey Veng province in Cambodia: a possible factor contributing to high iron stores in women. Journal of Water and Health, 13, 2.

7. British Geological Survey \& Department for Public Health Engineering, Government of the People's Republic of Bangladesh (2001) Arsenic Contamination of Groundwater in Bangladesh. BGS Technical Report WC/00/19 [DG Kinniburgh and PL Smedley, editors]. Keyworth: BGS.

8. Jaeggi T, Kortman GA, Moretti D, Chassard C, Holding P, Dostal A, Zimmermann MB, 2015. Iron fortification adversely affects the gut microbiome, increases pathogen abundance and induces intestinal inflammation in Kenyan infants. Gut, 64, 731-742.

9. Zimmermann MB, Chassard C, Rohner F et al.,2010. The effects of iron fortification on the gut microbiota in African children: a randomized controlled trial in Cote d'Ivoire. Am J Clin Nutr 92, 1406-1415.

10. Rahman S, Lee P, Raqib R, Roy AK, Khan MR, Ahmed F, 2019. Effect of Micronutrient Powder (MNP) with a Low-Dose of Iron on Hemoglobin and Iron Biomarkers, and Its Effect on Morbidities in Rural Bangladeshi Children Drinking Groundwater with a HighLevel of Iron: A Randomized Controlled Trial. Nutrients 11, 2756.

11. Saito H, 2014. Metabolism of iron stores. Nagoya J Med Sci 76, 235-254.

12. Graaf ID, 2016. EGU Blog: https://blogs.egu.eu/network/waterunderground/2016/12/20/limits-to-global-groundwater-use/ (accessed on 15 December 2019). 